\title{
Association between rs11001553 of $D K K 1$ and non-syndromic tooth agenesis in the Chinese Han population
}

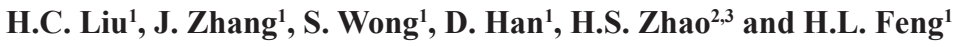 \\ ${ }^{1}$ Department of Prosthodontics, \\ Peking University School and Hospital of Stomatology, Beijing, China \\ ${ }^{2}$ Department of Medical Genetics, Peking University Health Science Center, \\ Beijing, China \\ ${ }^{3}$ Peking University Center for Human Disease Genomics, \\ Peking University Health Science Center, Beijing, China \\ Corresponding author: H.L. Feng \\ E-mail: kqfenghl@bjmu.edu.cn
}

Genet. Mol. Res. 13 (3): 7133-7139 (2014)

Received June 24, 2013

Accepted November 27, 2013

Published April 3, 2014

DOI http://dx.doi.org/10.4238/2014.April.3.4

\begin{abstract}
Tooth agenesis is one of the most common anomalies of human dentition. Recent genetic studies have provided information regarding a number of genes related to both syndromic and nonsyndromic forms of hypodontia. In a previous study, we found that polymorphism in rs 11001553 of $D K K 1$ was associated with hypodontia in the Chinese Han population. In this study, we extended this investigation to 89 individuals diagnosed with sporadic non-syndromic oligodontia (40 males and 49 females). These individuals were analyzed with 268 subjects (123 males and 145 females) diagnosed with nonsyndromic hypodontia and 190 healthy control subjects (99 males and 91 females). DNA was obtained from whole blood or saliva samples and genotyping was performed by matrix-assisted laser desorption ionization-time of flight mass spectrometry. Significant differences were observed in the allele and genotype frequencies of rs11001553 of
\end{abstract}


DKK1. These data demonstrated an association between rs11001553 of $D K K 1$, a tooth development-associated gene, and non-syndromic tooth agenesis in Chinese Han individuals. This information may provide further understanding of the molecular mechanisms of tooth agenesis. Furthermore, $D K K 1$ can be regarded as a marker gene for the risk of tooth agenesis.

Key words: Single nucleotide polymorphism; Tooth agenesis; Case-control study; DKK1

\section{INTRODUCTION}

Tooth agenesis is the most common dental developmental anomaly in humans, often representing a significant clinical problem (Nieminen, 2009; De Coster et al., 2009). The prevalence of dental agenesis of permanent teeth ranges from 2.2 to $10.1 \%$ in the general population excluding third molars (Polder et al., 2004). Tooth agenesis may present as part of a syndrome; however, the non-syndromic form is more common. Hypodontia is often used as a collective term to describe the absence of one to six teeth, excluding third molars (Vastardis, 2000; De Coster et al., 2009). Oligodontia refers to the absence of more than six teeth, excluding third molars (Song et al., 2009).

Several studies have indicated that tooth agenesis is mainly due to genetic factors (Vastardis, 2000; Thesleff, 2000, 2006; Nieminen, 2009; Mitsiadis and Luder, 2011; Galluccio et al., 2012). To date, mutations in PAX9 (paired box 9), MSX1 (msh homeobox 1), AXIN2 (axis inhibition protein 2), EDA, and WNT10A (wingless-type MMTV integration site family, member 10a) have been identified in non-syndromic tooth agenesis (Vastardis et al., 1996; Gerits et al., 2006; Han et al., 2008; Bergendal et al., 2011; Kantaputra and Sripathomsawat, 2011; van den Boogaard et al., 2012). However, mutations in these five genes cannot be identified in many cases. Furthermore, the etiological mechanism of non-syndromic tooth agenesis remains to be clarified.

Tooth development, which is a highly complicated process involving many genes and signaling pathways (Hu and Simmer, 2007), is regulated by tissue interactions and genetic networks, similar to other ectodermal organs. Any slight alterations in one or more of these genes may cause tooth agenesis. Gene polymorphism underlies the mechanism by which individuals exhibit variations within the extent of what is considered biologically normal. Furthermore, there is a close relationship between gene polymorphisms and susceptibility to dental developmental anomalies. Single nucleotide changes, which occur at a high frequency in the human genome, are the most common polymorphisms and may affect the function of genes. Therefore, single nucleotide polymorphisms (SNPs) may be risk factors for non-syndromic tooth agenesis.

In a previous study, we found that polymorphism in rs 11001553 of $D K K 1$ was associated with hypodontia in the Chinese Han population (Liu et al., 2012). DKK1 is a secreted protein with two cysteine-rich regions and is involved in embryonic development through its inhibition of the WNT signaling pathway (Fedi et al., 1999). Thus, mutations in DKK1 may affect tooth development via the WNT signaling pathway.

In this study, we extended the previous investigation to include 89 individuals (40 males and 49 females) diagnosed with sporadic non-syndromic oligodontia and conducted an 
analysis of these individuals with 268 subjects (123 males and 145 females) diagnosed with non-syndromic hypodontia and 190 healthy control subjects (99 males and 91 females). Our results confirmed that polymorphism in rs 11001553 of $D K K 1$ is associated with tooth agenesis in the Chinese Han population, especially in females.

\section{MATERIAL AND METHODS}

\section{Subject selection and sampling}

The details of 268 subjects (123 males and 145 females) diagnosed with sporadic nonsyndromic hypodontia (excluding third molars) and 190 healthy control subjects (99 males and 91 females) have been previously described (Liu et al., 2012). In this study, we included an additional 89 subjects (40 males and 49 females) diagnosed with sporadic non-syndromic oligodontia. All individuals participating in this study were genetically unrelated ethnic Han Chinese from Beijing or the surrounding regions.

None of the subjects had a history of tooth extraction or loss. Naturally missing teeth within the adult dentition were confirmed by X-ray examination and no other dental anomalies were observed in any of the subjects. All samples were obtained with informed written consent and blood samples or oral swabs were coded to maintain confidentiality. Genomic DNA was extracted from peripheral blood lymphocytes using the TIANamp Blood DNA kit (Tiangen, Beijing, China) and the TIANamp Swab DNA kit (Tiangen) according to manufacturer instructions. The extracted DNA samples were stored at $-20^{\circ} \mathrm{C}$ prior to analysis. This study was approved by the Institutional Review Board of Peking University School and Hospital of Stomatology.

\section{Polymorphism genotyping}

Primers for polymerase chain reaction and single-base extensions were designed using the Assay Designer software package (Sequenom, Inc., San Diego, CA, USA). SNP genotyping was performed by matrix-assisted laser desorption ionization-time of flight mass spectrometry (MALDI-TOF-MS) using the MassARRAY system (Sequenom) according to the instructions provided by the manufacturer. Completed genotyping reactions were spotted onto a 384-well spectroCHIP (Sequenom) using the MassARRAY system (Sequenom) and analyzed by MALDI-TOF-MS. Genotype calling was performed in real-time using the MassARRAY RT software version 3.0.0.4 and analyzed using the MassARRAY Typer software version 3.4 (Sequenom).

\section{Statistical analysis}

Hardy-Weinberg equilibrium was tested using a goodness-of-fit chi-square test to compare the observed genotype frequencies with the expected genotype frequencies among the control subjects. Clinical information and gender was compared across genotypes using chi-square tests. The associations between genotypes and the risk of tooth agenesis were estimated by computing the odds ratio $(\mathrm{OR})$ with $95 \%$ confidence intervals $(95 \% \mathrm{CI})$ from logistic regression analyses. $\mathrm{P}<0.05$ was considered to be statistically significant. All statistical tests for this analysis were performed using the SPSS 13.0 software. 


\section{RESULTS}

The general tooth agenesis case group was divided into two groups according to the number of missing teeth; the hypodontia group refers to the absence of one to six teeth and the oligodontia group refers to the absence of more than six teeth. We investigated the distribution of genotypes and alleles (Table 1). The CC and TT genotype frequencies were 82.5 and $0 \%$, respectively, in the hypodontia group, 86.5 and $0 \%$, respectively, in the oligodontia group and 74.2 and $2.1 \%$, respectively, in the control group. Significant differences were observed in the distribution of genotypes in the tooth agenesis cases (hypodontia $v s$ control, $\mathrm{P}=0.013$; oligodontia $v s$ control, $\mathrm{P}=0.047$; hypodontia + oligodontia vs control, $\mathrm{P}=0.011)$. Compared with the control group, significant differences in the combined CT and TT genotypes were also observed in the hypodontia group $(\mathrm{P}=0.033)$, oligodontia group $(\mathrm{P}=0.020)$ and the general tooth agenesis case group $(\mathrm{P}=0.010)$. Compared with an allele frequency of $13.9 \%$ in the control group, the frequency of the $\mathrm{T}$ allele was $8.8 \%$ in the hypodontia group and $6.7 \%$ in the oligodontia group (hypodontia $v s$ control, $\mathrm{P}=0.013$; oligodontia $v s$ control, $\mathrm{P}=0.013$; hypodontia + oligodontia $v s$ control, $\mathrm{P}=0.003)$.

Table 1. Genotype and allele frequencies of the rs 11001553 in hypodontic, oligodontic and normal individuals.

\begin{tabular}{|c|c|c|c|c|c|c|c|c|c|}
\hline & Hypodontia & Oligodontia & Control & $P_{\text {value }}{ }^{a}$ & Odds ratio $(95 \% \mathrm{CI})^{\mathrm{a}}$ & $P_{\text {value }}{ }^{b}$ & Odd ratio $(95 \% \mathrm{CI})^{\mathrm{b}}$ & $\mathrm{P}_{\text {value }}{ }^{\mathrm{C}}$ & Odds ratio $(95 \% \mathrm{CI})^{\circ}$ \\
\hline \multicolumn{10}{|l|}{ Genotype } \\
\hline $\mathrm{CC}$ & $221(82.5 \%)$ & $77(86.5 \%)$ & $141(74.2 \%)$ & & & & & & \\
\hline $\mathrm{CT}$ & $47(17.5 \%)$ & $12(13.5 \%)$ & $45(23.7 \%)$ & 0.013 & & 0.047 & & 0.011 & \\
\hline TT & $0(0 \%)$ & $0(0 \%)$ & $4(2.1 \%)$ & & & & & & \\
\hline $\mathrm{CT}+\mathrm{TT}$ & $47(17.5 \%)$ & $12(13.5 \%)$ & $49(25.8 \%)$ & 0.033 & $0.012(0.389-0.962)$ & 0.020 & $0.448(0.225-0.894)$ & 0.010 & $0.570(0.371-0.875)$ \\
\hline \multicolumn{10}{|l|}{ Allele } \\
\hline $\mathrm{C}$ & $489(91.2 \%)$ & $166(93.3 \%)$ & $327(86.1 \%)$ & & & & & & \\
\hline $\mathrm{T}$ & $47(8.8 \%)$ & $12(6.7 \%)$ & $53(13.9 \%)$ & 0.013 & $0.593(0.391-0.900)$ & 0.013 & $0.446(0.232-0.858)$ & 0.003 & $0.556(0.375-0.824)$ \\
\hline
\end{tabular}

We then investigated the relationship between gender and the distribution of genotypes and alleles by stratification of the case and control groups on the basis of gender. Significant differences in the frequencies of both alleles and genotypes were detected in comparisons of the female individuals in each of the groups (Table 2). The CT and TT genotype frequencies were lower in the female hypodontia group and female oligodontia group than in the male control group (hypodontia $v s$ control, $\mathrm{P}=0.016$; oligodontia $v s$ control, $\mathrm{P}=0.089$; hypodontia+oligodontia $v$ s control, $\mathrm{P}=0.004)$. Compared with the female control group, significant differences were also observed in the combined CT and TT genotypes in the female hypodontia group $(\mathrm{P}=0.021)$, the female oligodontia group $(\mathrm{P}=0.038)$ and the female general tooth agenesis case group $(\mathrm{P}=0.002)$. Furthermore, compared with an allele frequency of $15.4 \%$ in the female control group, the frequency of the $\mathrm{T}$ allele was $7.6 \%$ in the female hypodontia group and $6.1 \%$ in the female oligodontia group (hypodontia $v s$ control, $\mathrm{P}=0.007$; oligodontia $v s$ control, $\mathrm{P}=0.025$; hypodontia+oligodontia $v s$ control, $\mathrm{P}=0.002$ ). However, no statistically significant differences were detected in comparisons of the male groups (Table 3 ). 
Table 2. Genotype and allele frequencies of the rs11001553 in hypodontic, oligodontic and normal female individuals.

\begin{tabular}{|c|c|c|c|c|c|c|c|c|c|}
\hline & Hypodontia & Oligodontia & Control & P value ${ }^{a}$ & Odds ratio $(95 \% \mathrm{CI})^{\mathrm{a}}$ & $P$ value ${ }^{b}$ & Odds ratio $(95 \% \mathrm{CI})^{\mathrm{b}}$ & $P$ value ${ }^{c}$ & Odds ratio $(95 \% \mathrm{CI})^{\mathrm{c}}$ \\
\hline \multicolumn{10}{|l|}{ Genotype } \\
\hline $\mathrm{CC}$ & $123(84.8 \%)$ & $43(87.8 \%)$ & $66(72.5 \%)$ & & & & & & \\
\hline CT & $22(15.2 \%)$ & $6(12.2 \%)$ & $22(24.2 \%)$ & 0.016 & & 0.089 & & 0.004 & \\
\hline TT & $0(0 \%)$ & $0(0 \%)$ & $3(3.3 \%)$ & & & & & & \\
\hline $\mathrm{CT}+\mathrm{TT}$ & $22(15.2 \%)$ & $6(12.2 \%)$ & $25(27.5 \%)$ & 0.021 & $0.472(0.247-0.901)$ & 0.038 & $0.368(0.140-0.972)$ & 0.008 & $0.445(0.242-0.820)$ \\
\hline \multicolumn{10}{|l|}{ Allele } \\
\hline $\mathrm{C}$ & $268(92.4 \%)$ & $92(93.9 \%)$ & $154(84.6 \%)$ & & & & & & \\
\hline $\mathrm{T}$ & $22(7.6 \%)$ & $6(6.1 \%)$ & $28(15.4 \%)$ & 0.007 & $0.451(0.250-0.817)$ & 0.025 & $0.359(0.143-0.899)$ & 0.002 & $0.428(0.245-0.746)$ \\
\hline
\end{tabular}

${ }^{a}$ Hypodontia vs control; boligodontia vs control; chypodontia+oligodontia $v s$ control; $\mathrm{P}$ values lower than 0.05 are in bold type.

Table 3. Genotype and allele frequencies of the rs 11001553 in hypodontic, oligodontic and normal male individuals.

\begin{tabular}{|c|c|c|c|c|c|c|c|c|c|}
\hline & Hypodontia & Oligodontia & Control & Pvalue $^{a}$ & Odds ratio $(95 \% \mathrm{CI})^{\mathrm{a}}$ & P value ${ }^{b}$ & Odds ratio $(95 \% \mathrm{CI})^{\mathrm{b}}$ & P value ${ }^{c}$ & Odds ratio $(95 \% \mathrm{CI})^{\mathrm{c}}$ \\
\hline \multicolumn{10}{|l|}{ Genotype } \\
\hline $\mathrm{CC}$ & 98 (79.7\%) & $34(85.0 \%)$ & $75(75.8 \%)$ & & & & & & \\
\hline CT & $25(20.3 \%)$ & $6(15.0 \%)$ & $23(23.2 \%)$ & 0.547 & & 0.440 & & 0.303 & \\
\hline TT & $0(0)$ & $0(0)$ & $1(1.0 \%)$ & & & & & & \\
\hline $\mathrm{CT}+\mathrm{TT}$ & $25(20.3 \%)$ & $6(15.0 \%)$ & $24(24.2 \%)$ & 0.484 & $0.797(0.442-1.505)$ & 0.230 & $0.551(0.207-1.472)$ & 0.314 & $0.734(0.401-1.342)$ \\
\hline \multicolumn{10}{|l|}{ Allele } \\
\hline $\mathrm{C}$ & 221 (98.8\%) & $74(92.5 \%)$ & $173(87.4 \%)$ & & & & & & \\
\hline $\mathrm{T}$ & $25(10.2 \%)$ & $6(7.5 \%)$ & $25(12.6 \%)$ & 0.414 & $0.783(0.434-1.411)$ & 0.219 & $0.561(0.221-1.424)$ & 0.263 & $0.727(0.416-1.272)$ \\
\hline
\end{tabular}

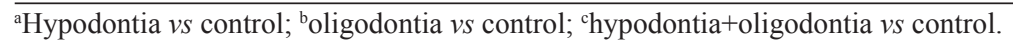

\section{DISCUSSION}

Many factors, including environmental and genetic factors, multi-agent chemotherapy and radiotherapy, may contribute to tooth agenesis (Vastardis, 2000). Although the exact mechanism of tooth agenesis has not been fully elucidated, genetic factors are believed to play a major role in this process. The incidence of tooth agenesis is extremely high, ranging from 2.2 to $10.1 \%$; however, only a few cases can be linked to precise genetic mutations, suggesting that tooth agenesis is a polygenic disorder. Hundreds of genes have been associated with tooth development and therefore potentially contribute to tooth agenesis. These genes code for signaling molecules, transcription factors, and factors controlling cell proliferation and differentiation (Thesleff, 2003). Individuals with distinct polymorphic alleles may exhibit subtle and specific phenotypic variations in dental patterning. Consequently, it can be speculated that association studies between gene polymorphisms and hypodontia, as well as other mild malformations, will reflect qualitative defects in embryogenesis (Opitz, 2000). Therefore, in this study, we focused on the association between tooth agenesis and SNPs.

In a previous study, we identified an association between two SNP markers (rs929387 of GLI3 and rs11001553 of DKK1) and non-syndromic hypodontia (Liu et al., 2012). In this study, we extended this investigation of rs11001553 to include non-syndromic oligodontic individuals (absence of more than six teeth). Statistical analysis of these groups confirmed our previous conclusion that polymorphism in rs11001553 of $D K K 1$ is a risk factor for nonsyndromic tooth agenesis in the Chinese Han population. We found that the difference was 
more significant in comparisons between the general tooth agenesis group (hypodontia and oligodontia) and the control group than between the hypodontia group and the control group. The data demonstrated a strong association between the marker rs 11001553 of DKK1 and nonsyndromic tooth agenesis in the Chinese Han population and implicated allele $\mathrm{C}$ as a risk factor.

Interestingly, following stratification of the case and control groups on the basis of gender, marked differences in the frequency of rs 11001553 were observed in comparisons between the stratified groups than between the case-control groups. Specifically, the differences were more significant in comparisons of the female case-control groups than between the unstratified case-control groups. Furthermore, no significant differences were detected in comparison of the male case-control groups. These data indicate that rs 11001553 is a risk factor for tooth agenesis in the female Chinese Han population. It should be noted that this study was conducted in a relatively small sample size, and it can be speculated that significant differences in male case-control comparisons could be revealed in a study conducted in a larger population size.

The rs11001553 (C/T) marker is located near the 5'-UTR of the DKK1 gene, which encodes a member of the dickkopf family. This secreted protein contains two cysteine-rich regions and is involved in embryonic development through its inhibition of the WNT signaling pathway (Fedi et al., 1999). DKK1 plays a significant role in tooth development. In the DKK1 transgenic mice, tooth development has been found to be arrested before the bud stage (Andl et al., 2002). DKK1 is an extracellular Wnt antagonist. Wnt signaling is essential for tooth development as indicated by transgenic mouse experiments (Mikkola and Millar, 2006). Mutations and polymorphisms in $A X I N 2$, an intracellular antagonist of Wnt signaling, have been associated with tooth agenesis (Lammi et al., 2004; Mostowska et al., 2006; Callahan et al., 2009). WNT10A mutations have also been reported in patients with tooth agenesis (Kantaputra and Sripathomsawat, 2011). It can be speculated that $D K K 1$ functions as an antagonist of the WNT signaling pathway, and that polymorphisms in $D K K 1$ may therefore affect tooth development.

Previous studies of polymorphisms and tooth agenesis are rare, with most focusing on PAX9, MSX1 and AXIN2 (Mostowska et al., 2006; Bianch et al., 2007; Callahan et al., 2009; Silva et al., 2009). Furthermore, only two studies have focused on the Chinese population (Pan et al., 2008; Liu et al., 2012). While syndromic tooth agenesis appears to be a monogenic disorder, unique mutations associated with non-syndromic tooth agenesis have not been identified, thus indicating that a polygenic mechanism underlies this form of disorder. Polymorphisms in tooth developmental genes may be a risk factor and the interaction between these risk factors may lead to tooth agenesis; however, this hypothesis needs to be confirmed in large sample size and multi-ethnic studies.

In summary, we further confirmed in this study an association between polymorphism in rs11001553 of $D K K 1$ and non-syndromic tooth agenesis in the Chinese Han population, especially in females. Functional studies are required to elucidate the mechanism of this association. In the future, extensive screening of more genetic variants of the $D K K 1$ gene, from larger size samples, will be required to confirm that $D K K 1$ is a candidate genetic marker of non-syndromic tooth agenesis.

\section{ACKNOWLEDGMENTS}

Research supported by the Beijing Natural Science Foundation (\#7092113), the National Natural Science Foundation (\#81070814 and \#81100725), and the Capital Medical Developing Foundation (\#2007-1005). We thank the subjects and their families for their participation. 


\section{REFERENCES}

Andl T, Reddy ST, Gaddapara T and Millar SE (2002). WNT signals are required for the initiation of hair follicle development. Dev. Cell 2: 643-653.

Bergendal B, Klar J, Stecksen-Blicks C, Norderyd J, et al. (2011). Isolated oligodontia associated with mutations in EDARADD, AXIN2, MSX1, and PAX9 genes. Am. J. Med. Genet. A 155A: 1616-1622.

Bianch FJ, de Oliveira TF, Saito CB, Peres RC, et al. (2007). Association between polymorphism in the promoter region (G/C-915) of $P A X 9$ gene and third molar agenesis. J. Appl. Oral. Sci. 15: 382-386.

Callahan N, Modesto A, Meira R, Seymen F, et al. (2009). Axis inhibition protein 2 (AXIN2) polymorphisms and tooth agenesis. Arch. Oral Biol. 54: 45-49.

De Coster PJ, Marks LA, Martens LC and Huysseune A (2009). Dental agenesis: genetic and clinical perspectives. J. Oral Pathol. Med. 38: 1-17.

Fedi P, Bafico A, Nieto SA, Burgess WH, et al. (1999). Isolation and biochemical characterization of the human Dkk-1 homologue, a novel inhibitor of mammalian Wnt signaling. J. Biol. Chem. 274: 19465-19472.

Galluccio G, Castellano M and La Monaca C (2012). Genetic basis of non-syndromic anomalies of human tooth number. Arch. Oral Biol. 57: 918-930.

Gerits A, Nieminen P, De Muynck S and Carels C (2006). Exclusion of coding region mutations in MSX1, PAX9 and AXIN2 in eight patients with severe oligodontia phenotype. Orthod. Craniofac. Res 9: 129-136.

Han D, Gong Y, Wu H, Zhang X, et al. (2008). Novel EDA mutation resulting in X-linked non-syndromic hypodontia and the pattern of EDA-associated isolated tooth agenesis. Eur. J. Med. Genet. 51: 536-546.

Hu JC and Simmer JP (2007). Developmental biology and genetics of dental malformations. Orthod. Craniofac. Res. 10: 45-52.

Kantaputra P and Sripathomsawat W (2011). WNT10A and isolated hypodontia. Am. J. Med. Genet. A 155A: 1119-1122.

Lammi L, Arte S, Somer M, Jarvinen H, et al. (2004). Mutations in AXIN2 cause familial tooth agenesis and predispose to colorectal cancer. Am. J. Hum. Genet. 74: 1043-1050.

Liu H, Zhang J, Song S, Zhao H, et al. (2012). A case-control study of the association between tooth-development gene polymorphisms and non-syndromic hypodontia in the Chinese Han population. Eur. J. Oral Sci. 120: 378-385.

Mikkola ML and Millar SE (2006). The mammary bud as a skin appendage: unique and shared aspects of development. $J$. Mammary. Gland Biol. Neoplasia 11: 187-203.

Mitsiadis TA and Luder HU (2011). Genetic basis for tooth malformations: from mice to men and back again. Clin. Genet. 80: 319-329.

Mostowska A, Biedziak B and Jagodzinski PP (2006). Axis inhibition protein 2 (AXIN2) polymorphisms may be a risk factor for selective tooth agenesis. J. Hum. Genet. 51: 262-266.

Nieminen P (2009). Genetic basis of tooth agenesis. J. Exp. Zool. B Mol. Dev. Evol. 312B: 320-342.

Opitz JM (2000). Heterogeneity and minor anomalies. Am. J. Med. Genet. 92: 373-375.

Pan Y, Wang L, Ma J, Zhang W, et al. (2008). PAX9 polymorphisms and susceptibility to sporadic tooth agenesis: a casecontrol study in southeast China. Eur. J. Oral Sci. 116: 98-103.

Polder BJ, Van't Hof MA, Van der Linden FP and Kuijpers-Jagtman AM (2004). A meta-analysis of the prevalence of dental agenesis of permanent teeth. Community Dent. Oral Epidemiol. 32: 217-226.

Silva ER, Reis-Filho CR, Napimoga MH and Alves JB (2009). Polymorphism in the Msx I gene associated with hypodontia in a Brazilian family. J. Oral Sci. 51: 341-345.

Song S, Han D, Qu H, Gong Y, et al. (2009). EDA gene mutations underlie non-syndromic oligodontia. J. Dent. Res. 88: 126-131.

Thesleff I (2000). Genetic basis of tooth development and dental defects. Acta Odontol. Scand. 58: 191-194.

Thesleff I (2003). Epithelial-mesenchymal signalling regulating tooth morphogenesis. J. Cell Sci. 116: 1647-1648.

Thesleff I (2006). The genetic basis of tooth development and dental defects. Am. J. Med. Genet. A 140: 2530-2535.

van den Boogaard MJ, Creton M, Bronkhorst Y, van der Hout A, et al. (2012). Mutations in WNT10A are present in more than half of isolated hypodontia cases. J. Med. Genet. 49: 327-331.

Vastardis H (2000). The genetics of human tooth agenesis: new discoveries for understanding dental anomalies. Am. $J$. Orthod. Dentofacial Orthop. 117: 650-656.

Vastardis H, Karimbux N, Guthua SW, Seidman JG, et al. (1996). A human MSX1 homeodomain missense mutation causes selective tooth agenesis. Nat. Genet. 13: 417-421. 